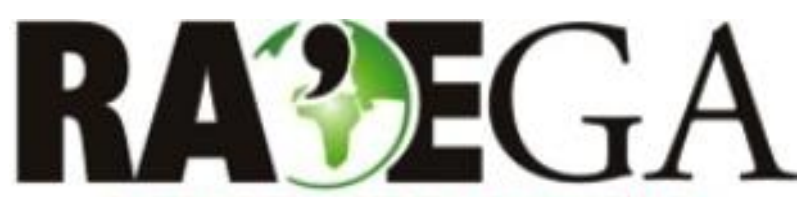

O ESPAÇO GEOGRÁFICO EM ANÁLISE

\title{
AVALIAÇÃO MACRO E MICROESTRUTURAL DE SOLOS SOB DIFERENTES USOS A PARTIR DE ANÁLISES FÍSICAS, MICROSCOPIA ELETRÔNICA E ESPECTROSCOPIA POR ENERGIA DISPERSIVA
}

\section{MACRO AND MICROSTRUCTURAL EVALUATION OF SOILS UNDER DIFFERENT USES THROUGH PHYSICAL ANALYSES, ELETRONIC MICROSCOPY AND DISPERSIVE ENERGY SPECTROSCOPY}

\author{
Raphael Rodrigues Brizzi ${ }^{1}$, Hugo Portocarrero ${ }^{2}$, Andréa Paula de Souza ${ }^{3}$, Alexander Josef Sá Tobias da \\ Costa $^{4}$, Luana de Almeida Rangel ${ }^{5}$
}

\section{RESUMO}

A degradação das propriedades físicas e químicas dos solos de acordo com o uso e o manejo das terras tem sido alvo de muita preocupação para agricultores e gestores devido à questão dos processos erosivos. Nessa perspectiva, o presente estudo teve por objetivo subsidiar e aprofundar o entendimento sobre relações entre a estruturação dos solos a nível microscópico e parâmetros macroscópicos de agregação a partir de análises físicas, microscopia eletrônica de varredura e espectroscopia por energia dispersiva. Para tal, foram selecionados dois perfis de solos sob dois diferentes tipos de usos: fragmento florestal e pousio. Em campo, além da coleta de amostras, foram realizadas as descrições dos perfis de solos e as análises morfológicas, e posteriormente, em laboratório, foram realizadas análises granulométricas, de estabilidade dos agregados em água (DMG, DMP e IEA), determinação do teor carbono orgânico, análise estrutural no Microscópio Eletrônico de Varredura - MEV, além da identificação dos elementos químicos presentes através da Espectrometria por Energia Dispersiva - EDS. As imagens obtidas no MEV demonstraram ser o uso florestal mais correlacionado à boa microagregação dos horizontes superficiais dos solos avaliados, enquanto que o solo sob pousio apresentou uma microestrutura cimentada sugerindo forte antropização do horizonte, possivelmente por pé de arado. Os resultados deste trabalho evidenciaram o auxílio fornecido pela ferramenta MEV na compreensão dos processos de agregação, sobretudo em relação a microagregação, possibilitando uma discussão mais ampla acerca do efeito de diferentes práticas de manejo e conservação dos solos em seus estados de agregação.

Palavras-chave: Agregação, Estabilidade de agregados; Uso do solo; Microscópio Eletrônico de Varredura; Espectrometria por Energia Dispersiva.

\section{ABSTRACT}

The degradation of the physical and chemical properties of soils according to land use and management has been a subject of great concern for farmers and managers due to erosion processes. In this perspective, the present study had as objective to subsidize and to deepen the understanding on relations between the soil structure at the microscopic level and macroscopic parameters of aggregation from physical analyzes, scanning electron microscopy and dispersive energy spectroscopy. For this, two soil profiles were selected under two different types of uses: forest fragment and fallow. In the field, in addition to the sample collection, soil profile descriptions and morphological analyzes were performed, and later, in the laboratory, granulometric analyzes, aggregate stability in water (DMG, DMP and IEA), determination of the content organic carbon, structural analysis in the Scanning Electron Microscope - SEM, besides the identification of the chemical elements present through the Energy Dispersive Spectrometry - EDS. The images obtained in the SEM showed that soil under forest was the most correlated to the good microaggregation of the superficial horizons of the evaluated soils, while fallow presented a cemented microstructure suggesting a strong anthropization of the horizon, possibly by a plow. The results of this work showed the help provided by the MEV tool in the understanding of aggregation processes, especially in relation to microaggregation, allowing a broader discussion about the effect of different management practices and soil conservation in their states of aggregation.

Key-words: Aggregation, Aggregate Stability; soil land use; Scanning Electron Microscopy; Energy Dispersive Spectrometry.

Recebido em: 19/05/2017 Aceito em: 20/02/2019

\footnotetext{
${ }^{1}$ Instituto Federal do Rio de Janeiro, Arraial do Cabo/RJ, e-mail: raphael.brizzi@ifrj.edu.br

${ }^{2}$ Universidade do Estado do Rio de Janeiro, Rio de Janeiro/RJ, e-mail: hportocarrero@gmail.com

${ }^{3}$ Faculdade de Educação da Baixada Fluminense, Duque de Caxias/RJ, e-mail: andreaps.uerj@gmail.com

${ }^{4}$ Universidade do Estado do Rio de Janeiro, Rio de Janeiro/RJ, e-mail: ajcostageo@gmail.com

${ }^{5}$ Universidade Federal do Rio de Janeiro, Rio de Janeiro/RJ, e-mail: luarangel@ufrj.br
} 


\section{AVALIAÇÃO MACRO E MICROESTRUTURAL DE SOLOS SOB DIFERENTES USOS A PARTIR DE ANÁLISES FÍSICAS, MICROSCOPIA ELETRÔNICA E ESPECTROSCOPIA}

\section{INTRODUÇÃO}

A degradação das propriedades físicas e químicas dos solos de acordo com o uso e o manejo das terras tem sido alvo de muita preocupação para agricultores e gestores devido à questão dos processos erosivos. Seus efeitos são visíveis nas encostas e causa prejuízos consideráveis, como a perda dos horizontes superficiais dos solos e de suas fertilidades.

Assim, usos do solo como o cultivo convencional e as pastagens tendem a alterar a estrutura do solo e aumentar a suscetibilidade à erosão (BERTONI, LOMBARDI NETTO, 2010), principalmente pelas formas de manejo os quais the são inerentes, como a prática da queima - que deixa o solo exposto às gotas de chuva e elimina a fauna e a flora superficial do solo, e a utilização de maquinário e da pecuária extensiva - que aumenta a densidade e destroem os macroagregados do horizonte superficial. Tais práticas refletem numa diminuição drástica da infiltração e no aumento do escoamento superficial que, consequentemente, dá início a erosão laminar que pode evoluir para a formação de ravinas e voçorocas nas encostas (HORTON, 1945; COELHO NETTO, 2009).

Em contrapartida, Puig (2008) destaca a importância da floresta tropical nas trocas de energia e matéria, ciclagem de nutrientes, trocas gasosas, regulagem térmica com a camada de serapilheira e sua decomposição, manutenção da fauna endopedônica e no aumento da porosidade e da infiltração pela maior presença de raízes, sendo Tisdall e Oades (1982), Carter (2001) e Shukla et al. (2006) destacando ser a matéria orgânica o melhor indicador da estruturação dos solos, e a estabilidade dos agregados como parâmetro de agregação para resistência à erosão (YODER, 1936).

Diversos autores (WENDLIN et al.,2005; AN et al.,2010; DENEF et al., 2001; SANTOS, et al., 2002, GROSBELLET et al., 2011) destacaram que o uso florestal correlaciona-se a maiores valores de macroagregados, resultando em uma maior estabilização do carbono orgânico e resistência à erosão. Entretanto, autores recentes têm divergido da ideia de que a floresta apresenta sempre os melhores índices de agregação dentro de uma região onde exista uso agropecuário.

Dois exemplos de trabalhos aonde se chegou a estes resultados foram os de Demarchi et al. (2011) e Mendes et al. (2011), que mostraram que os índices de estabilidade dos agregados foram maiores no uso do solo sob cultivo de banana e pastagem quando comparados ao uso florestal, mesmo tendo alguns autores (JONHSON, 1992, CENTRINI, 2005, KNICKER, 2007, BERTOL et al., 2011) destacando os efeitos da prática da queimada nas propriedades dos solos e no aumento da erosão.

Portanto, não existe uma correlação trivial entre uso florestal e uma melhor agregação do solo nos horizontes superficiais em relação aos demais usos, mesmo quando sob histórico de manejo tradicional (queima e corte), sendo este um pressuposto desta pesquisa.

Nessa perspectiva, o presente estudo teve por objetivo subsidiar e aprofundar o entendimento sobre relações entre a estruturação dos solos a nível microscópico e parâmetros macroscópicos de agregação, a partir de análises físicas, microscopia eletrônica de varredura (MEV) e espectroscopia por energia dispersiva (EDS). Para tal, foram selecionados dois perfis de solos sob dois diferentes tipos de usos: fragmento florestal e pousio. Acredita-se que o MEV pode auxiliar na avaliação microestrutural do solo, possibilitando a observação e análise de agentes cimentantes, da composição básica intra-agregados, da superfície dos agregados ou em revestimentos - coatings, assim como, avanços em relação a estudos sobre efeitos do manejo e da conservação dos solos.

\section{MATERIAIS E MÉTODOS}

O presente estudo foi realizado em uma encosta situada (figura 1) dentro da bacia 


\section{AVALIAÇÃO MACRO E MICROESTRUTURAL DE SOLOS SOB DIFERENTES USOS A PARTIR DE ANÁLISES FÍSICAS, MICROSCOPIA ELETRÔNICA E ESPECTROSCOPIA}

hidrográfica do Córrego da Caçada (BHCC), pertencente à Área de Proteção Ambiental Federal de Cairuçu, no município de Paraty-RJ, sob as coordenadas UTM 7829000N, 7424000S, 527000 e $532000 W$ - fuso 23 Sul. Nesta encosta foram selecionados dois locais para a realização dos estudos: um fragmento florestal de Mata Atlântica e uma pastagem abandonada. Foram abertos perfis de solos e realizadas as análises morfológicas para cada uso. Posteriormente foi realizada a coleta das amostras dos horizontes superficiais para a realização das avaliações em laboratório. A localização da área, bem como destes dois pontos, encontra-se na Figura 1.

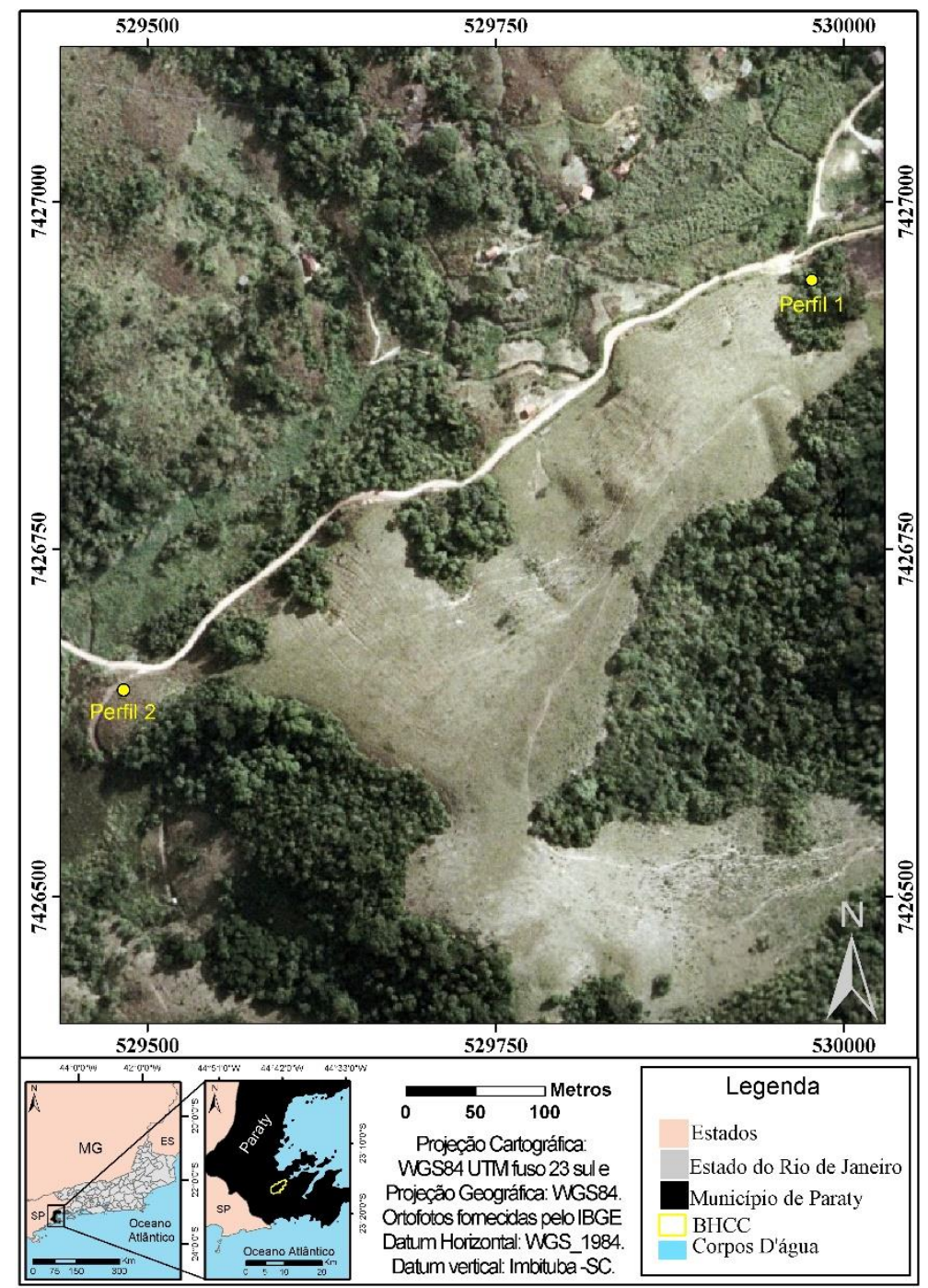

Figura 01 - Localização da área de estudo destacando os perfis estudados.

De acordo o ICMBio (2004), a área de estudo apresenta clima do tipo tropical úmido/superúmido, correspondente à classificação Cwa de KOPPEN. É influenciado principalmente pelos altos regimes pluviométricos, temperatura e relevo. A série histórica dos dados de precipitação indica uma média de $1.547 \mathrm{~mm}$ de chuva/ano, apresentando índices que variam de 768 a $2.150 \mathrm{~mm}$, sendo a média mensal de chuvas de aproximadamente $129 \mathrm{~mm}$, e com temperaturas que variam de 21 a $23^{\circ} \mathrm{C}$.

O embasamento cristalino da BHCC, constituído principalmente por formações granito-gnáissicas, é resultado da colisão brasiliana, pertencente à era Proterozóica - 


\section{AVALIAÇÃO MACRO E MICROESTRUTURAL DE SOLOS SOB DIFERENTES USOS A PARTIR DE ANÁLISES FÍSICAS, MICROSCOPIA ELETRÔNICA E ESPECTROSCOPIA}

Neoproterozoico (650-545 Ma), que originou o soerguimento do relevo da Serra do Mar, caracterizando um relevo de Horst e Graben (FERRARI, 2001; HASUI, 2010), onde sofreram sucessivos processos abrasivos ao longo das eras geológicas, que possibilitou a formação de patamares escalonados com presença significativa dos depósitos de tálus e antigos depósitos oriundos de corridas de massa nas encostas. A geomorfologia é marcada pela presença de montanhas e morros, cones de dejeção e corpos de tálus (derivados de depósitos de corridas de massa pretéritos) e planície fluvio-marinha na sua jusante. Segundo Bigarella e Mousinho (1965), o tipo de geomorfologia encontrada na bacia hidrográfica do Córrego da Caçada favorece a formação de colúvios no terço médio e inferior das encostas, até que este material chegue às calhas fluviais (alúvio). Segundo o mapa de reconhecimento da Embrapa (2003), na BHCC podem ser encontrados Cambissolo álico e Latossolo Vermelho-Amarelo álico, com declividades acentuadas que corroboram para o processo erosivo e inviabilizam o uso de maquinário nas encostas. Ao longo de toda a encosta em avaliação foram observados somente os Cambissolos, que são os objetos, portanto, das avaliações no decorrer deste artigo.

Para as análises de solos foram coletadas amostras indeformadas e amolgadas seguindo-se a metodologia adotada no Manual de Descrição e Coleta de Solo no Campo, da Sociedade Brasileira de Ciência dos Solos (SANTOS et al., 2005). As amostras amolgadas foram utilizadas para as análises morfológicas e de distribuição granulométrica, e as indeformadas foram coletadas para as análises de estabilidade de agregados em água pelo método proposto de Yoder (1936), modificado por Castro Filho et al. (1998, 2002). Os blocos de solo foram coletados na profundidade de 0 $10 \mathrm{~cm}$, com três repetições, possibilitando a realização de análises estatísticas.
Para a execução do ensaio de Yoder os blocos foram suavemente quebrados e homogeneizados com peneiras de $4 \mathrm{~mm}$ e 2 $\mathrm{mm}$, antes do tamisamento úmido, para ser determinada a distribuição das classes (2,0 $1,0-0,5-0,25-0,125$ e $<0,125 \mathrm{~mm})$ de agregados por via úmida. As amostras oscilaram durante 15 minutos, com aproximadamente 32 rotações por minuto (EMBRAPA, 2011). Após o término das oscilações, o conteúdo retido em cada uma das peneiras foi secado em estufa a $105^{\circ} \mathrm{C}$ durante 24 horas. Os valores obtidos nos peneiramentos foram usados para cálculo de três parâmetros utilizando-se das equações de modificadas de Kemper e Rosenau (1986). O Diâmetro Médio Geométrico (DMG) é uma estimativa do tamanho médio dos agregados que mais ocorrem no solo. O Diâmetro Médio Ponderado (DMP) é tanto maior quanto maior for a porcentagem de agregados grandes retidos nas peneiras com malhas maiores. 0 Índice de Estabilidade de Agregados (IEA) é uma medida da agregação total, mas sem considerar as classes de distribuição de tamanho dos agregados dos índices de estabilidade. O DMP é determinado através da equação abaixo, em que $\mathrm{W}_{\mathrm{i}}=$ proporção (\%) de cada classe em relação ao total; e $\mathrm{X}_{\mathrm{i}}=$ diâmetro médio das classes, expresso em $\mathrm{mm}$ :

$$
D M P(m m)=\sum_{i=1}^{n}\left(x_{i} \cdot w_{i}\right)
$$

O DMG é determinado através da equação abaixo, em que $\mathrm{W}_{\mathrm{i}}=$ proporção (\%) de cada classe em relação ao total; e $\mathrm{X}_{\mathrm{i}}=$ diâmetro médio das classes, expresso em $\mathrm{mm}$ :

$$
D M G(m m)=\exp \frac{\sum w_{i} \ln x_{i}}{\sum w_{i}}
$$

O IEA é determinado através da equação abaixo, em que o índice é expresso em porcentagem: 


\section{AVALIAÇÃO MACRO E MICROESTRUTURAL DE SOLOS SOB DIFERENTES USOS A PARTIR DE ANÁLISES FÍSICAS, MICROSCOPIA ELETRÔNICA E ESPECTROSCOPIA}

$$
I E A=\frac{\text { peso total dos agregados }- \text { peso dos agregados }<0,25 \mathrm{~mm}}{\text { peso da amostra seca }} \times 100
$$

O teste estatístico utilizado para comparar os diferentes usos foi o KruskalWallis. Esse teste não paramétrico é utilizado para comparar três ou mais amostras independentes, e indica se há diferença entre pelo menos duas delas (TRIOLA, 2008). Para realização do teste foi utilizado o software livre Action versão 2.5 disponível no site Portal Action.

Foram separados os agregados retidos nas peneiras para analisar o teor de carbono orgânico por titulometria via Dicromato de Potássio (EMBRAPA, 2011). As análises foram realizadas em duplicada para cada peneira do conjunto, totalizando quatro análises por classe de agregado para fins estatísticos.

Para as análises microestruturais no Microscópio Eletrônico de Varredura (MEV), foram coletados torrões dos horizontes superficiais, onde foi selecionado um agregado (previamente seco ao ar) de aproximadamente $2 \mathrm{~cm}^{3}$, submetido à emissão de feixes de elétrons retroespalhados em câmara de baixo vácuo, sendo possível a ampliação máxima em até 300.000 vezes. Ao MEV, estava acoplado o espectrômetro de energia dispersiva (EDS) onde foi possível a identificação mineralógica através da emissão contínua de Raios - $X$, comumente distribuídos do baixo número atômico (baixa energia) para elevado (alta energia). Nos gráficos obtidos o eixo " $Y$ " representa a frequência dos elementos químicos e o eixo " $X$ " a energia gasta para a sua identificação (MALISKA, 2000).

\section{RESULTADOS E DISCUSSÃO}

Os resultados das análises morfológicas e granulométricas encontram-se sintetizados na Tabela 1 . Nota-se que há uma diferença significativa de espessurado horizonte superficial de solo sob fragmento florestal em relação ao pousio, apresentando mais do que o dobro de espessura do primeiro para o segundo, respectivamente.

A consistência encontrada no horizonte superficial do solo sob fragmento florestal é típica de solos argilosos, apresentando coesão moderada, raízes médias e atividade biológica de formigas e minhocas, tendo sido constatada em campo forte bioturbação.

Quanto ao solo sob pousio, apresentou horizonte superficial com estrutura de tamanho pequeno e grau moderado, consistência dura quando seca, friável quando úmida e ligeiramente plástico e pegajoso quando molhado. A coesão é forte, com raízes médias e atividade biológica ausente. 
Brizzi et al.

\section{AVALIAÇÃO MACRO E MICROESTRUTURAL DE SOLOS SOB DIFERENTES USOS A PARTIR DE ANÁLISES FÍSICAS, MICROSCOPIA ELETRÔNICA E ESPECTROSCOPIA}

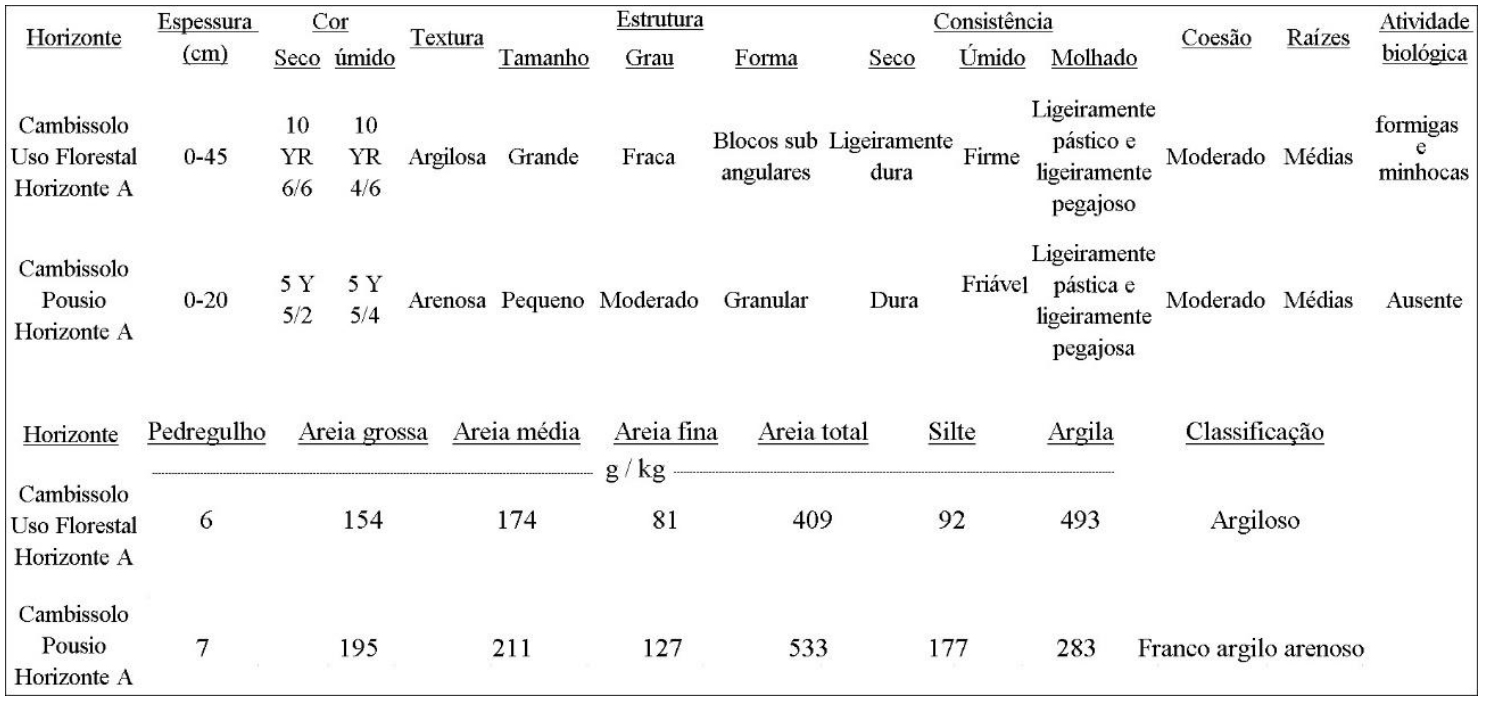

Tabela 01 - Análise morfológica e da distribuição granulométrica do horizonte superficial do Cambissolo sob uso florestal e pousio.

Segundo Kiehl (1979) solos argilosos possuem maior aglutinação entre as partículas, quando se comparado com um solo mais arenoso. Isso é explicado pela maior capacidade de troca catiônica e a forma dos argilominerais, que possibilita a formação de solos mais coesos, com estrutura forte e grande. Entretanto, deve-se destacar que tais características são influenciadas pela mineralogia das argilas, podendo haver solos argilosos com características e adesão e coesão diferenciadas.

A profundidade dos horizontes (tabela 1) possibilita inferir que o uso do solo sob pousio está tendo maior perda de solo pela erosão, agravado pela sua distribuição granulométrica franco-argilo-arenosa, com maior porcentagem de areia fina e silte, sendo este intervalo granulométrico altamente suscetível à erosão (MORGAN, 1995). Assim, além das formas de manejo, a agregação também está associada à textura do solo (BRONICK; LAL, 2005).

O uso do solo sob floresta, neste caso, influencia a formação de uma estrutura grande e com blocos subangulares, o que possivelmente acarretará em maior infiltração da água no solo diante de um evento chuvoso. Já no pousio, o peso do gado e a prática da queimada aumentam a ação das gotas de chuva na superfície do solo, justificando a classificação granulométrica mais grosseira, pois o silte e a argila são lixiviados e mantém a forma da estrutura granular. Nota-se também que o uso da queimada influencia diretamente na redução da atividade biológica, sendo esta fundamental na formação de macroagregados, onde no uso do pousio ela é ausente.

Nas análises de estabilidade dos agregados em água (figura 2), a floresta apresentou índices de agregação mais baixos do que no pousio. Enquanto o DMG foi de $0,91 \mathrm{~mm}$ na floresta, no pousio foi de $1,62 \mathrm{~mm}$. Já o DMP foi de 1,97 na floresta e de 2,28 no pousio. Porém, somente o DMG apresentou diferença significativa entre os usos pelo teste de Kruskal-Wallis $(p<0,05)$. Já para o DMP não foi constatada diferença. 


\section{AVALIAÇÃO MACRO E MICROESTRUTURAL DE SOLOS SOB DIFERENTES USOS A PARTIR DE ANÁLISES FÍSICAS, MICROSCOPIA ELETRÔNICA E ESPECTROSCOPIA}

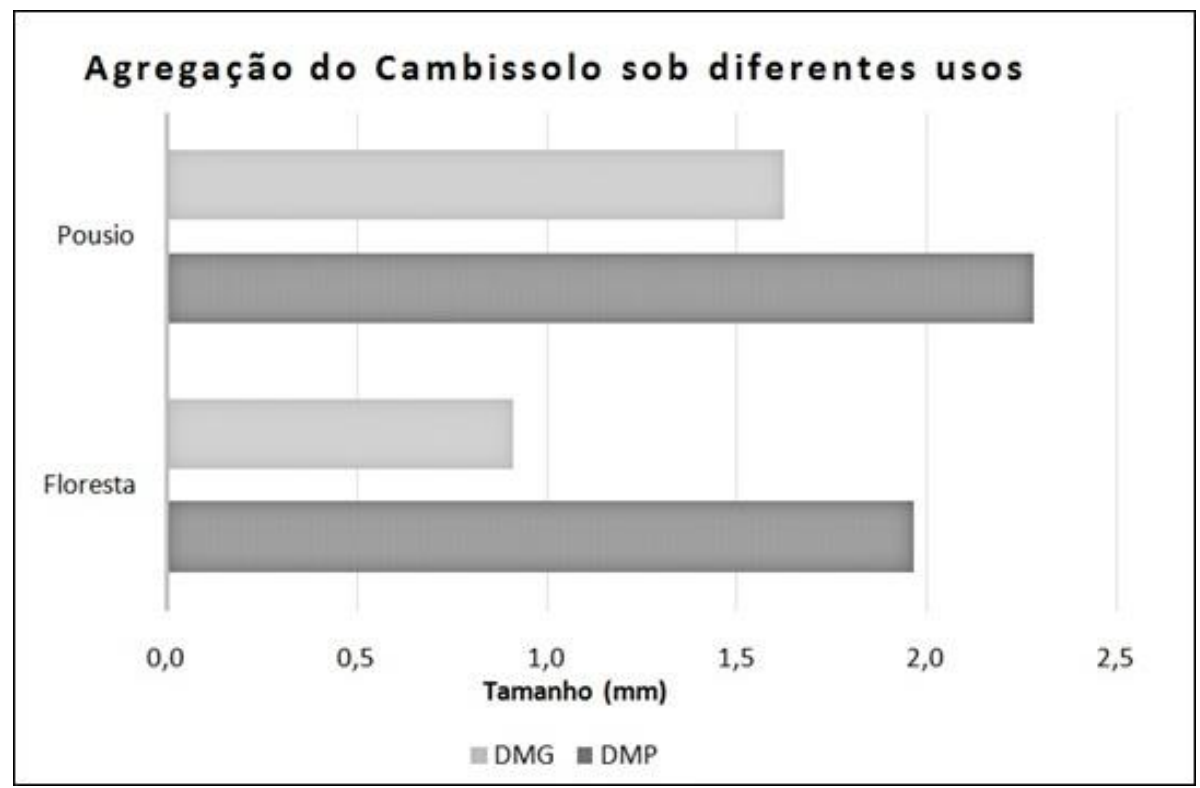

Figura 02 - Agregação do horizonte superficial do Cambissolo sob diferentes usos.

Apenas o DMG da floresta apresentou valores de agregação inferiores aos considerados aceitáveis (>1 $\mathrm{mm}$ ) por diversos autores (FATTET et al., 2011; CASTRO FILHO et al., 2002). O pousio apresentou os maiores valores de DMP e DMG, revelando taxas de agregação melhores do que da área de floresta, o que resulta diretamente num maior índice de estabilidade dos agregados - IEA. Isto pode estar relacionado à baixa utilização das terras para plantio, à textura do solo e às raízes das gramíneas (SILVA; MIELNICZUK, 1997, 1998).

É importante salientar que em geral em florestas ocorre uma sucessão mais gradual em todo o ecossistema, que é mais equilibrado. Neste sentido, a vegetação perenifólia/subperenifólia nas florestas propicia uma deposição de serrapilheira mais lenta em relação ao pousio, onde predominam gramíneas de rápido crescimento com elevada produção de material formador de serapilheira. Isto é, na área de pousio ocorrem processos de ciclagem mais rápidos, o que não implica em um acréscimo na resiliência destes ambientes.

$$
\text { Além de avaliar os índices de }
$$
agregação, é importante analisar a porcentagem de macro e micro agregados (figura 3), que também reflete na qualidade do solo e na sua estabilidade. Os microagregados do solo segundo Denef et al. (2001) são compostos por agregados de diâmetro inferior a $0,5 \mathrm{~mm}$. Quando os macroagregados do solo estão pouco estáveis, isto é, quando os agentes cimentantes - argila, metais polivalentes e matéria orgânica - não conseguem unir as partículas de solo, pode haver desagregação e formação de microagregados. Entretanto todo o processo de agregação dos solos inicia-se evidentemente a nível microestrutural, o que indica a importância da avaliação em ambas as escalas, isto é, tanto em nível macroestrutural, onde são avaliados os macroagregados, quanto em nível microestrutural, onde são avaliados microagregados. 


\section{AVALIAÇÃO MACRO E MICROESTRUTURAL DE SOLOS SOB DIFERENTES USOS A PARTIR DE ANÁLISES FÍSICAS, MICROSCOPIA ELETRÔNICA E ESPECTROSCOPIA}

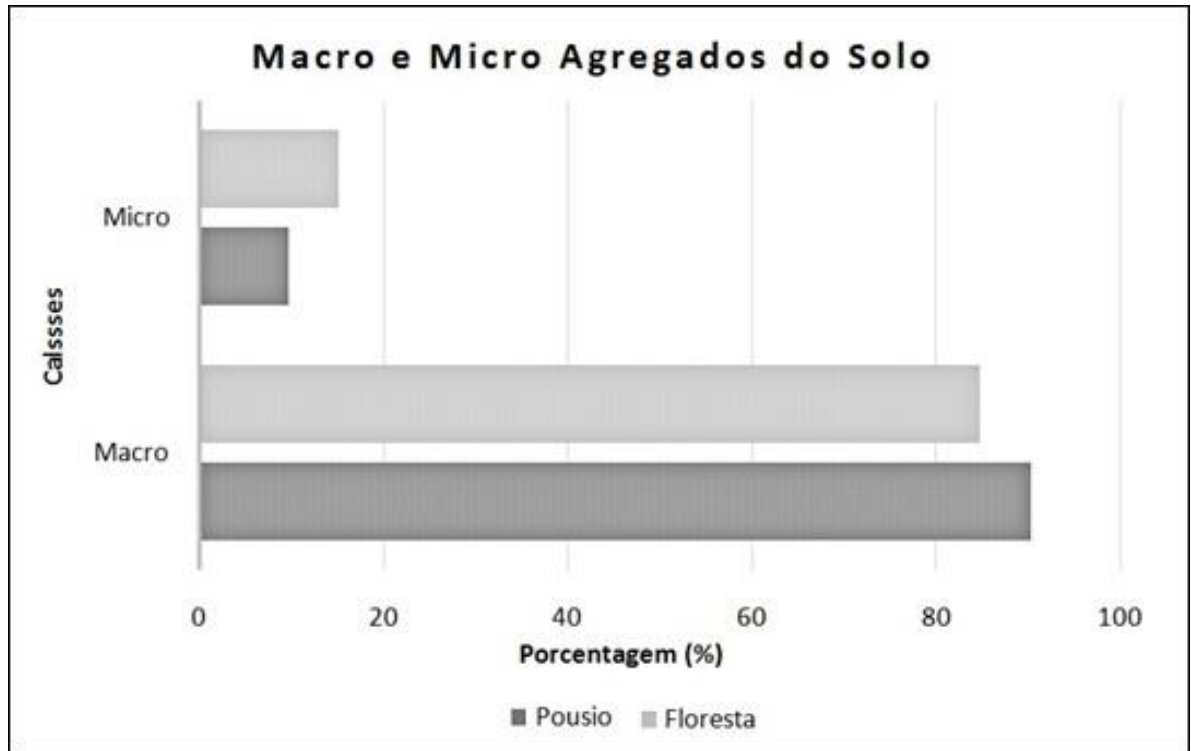

Figura 03 - Distribuição de micro e macro agregados do horizonte superficial do solo sob diferentes usos. As duas classes de distribuição apresentaram diferença significativa entre os usos de acordo com o teste de Kruskal-Wallis $(p<0,05)$.

Observa-se na figura 3 que os valores evidenciam boa agregação do solo (FATTET et al., 2011), com a área de pousio apresentando menor porcentagem de microagregados $(9,73 \%)$ e a área de floresta com uma maior porcentagem (15,17\%). Seguindo a tendência dos índices de agregação, entretanto, a área de pousio apresentou maior porcentagem de macroagregados $(90,27 \%)$, enquanto a área de floresta apresentou menor porcentagem $(84,83 \%)$.
Tais resultados podem estar relacionados com o estoque de carbono orgânico, que evidencia no pousio, valores superiores a 10 g.kg-1, enquanto que no fragmento florestal o estoque foi inferior a 10 g.kg-1. A diferença significativa pelo teste de Kruskal-Wallis $(p<0,05)$ foi constatada entre os usos em todas as classes de peneira analisadas (figura 4).

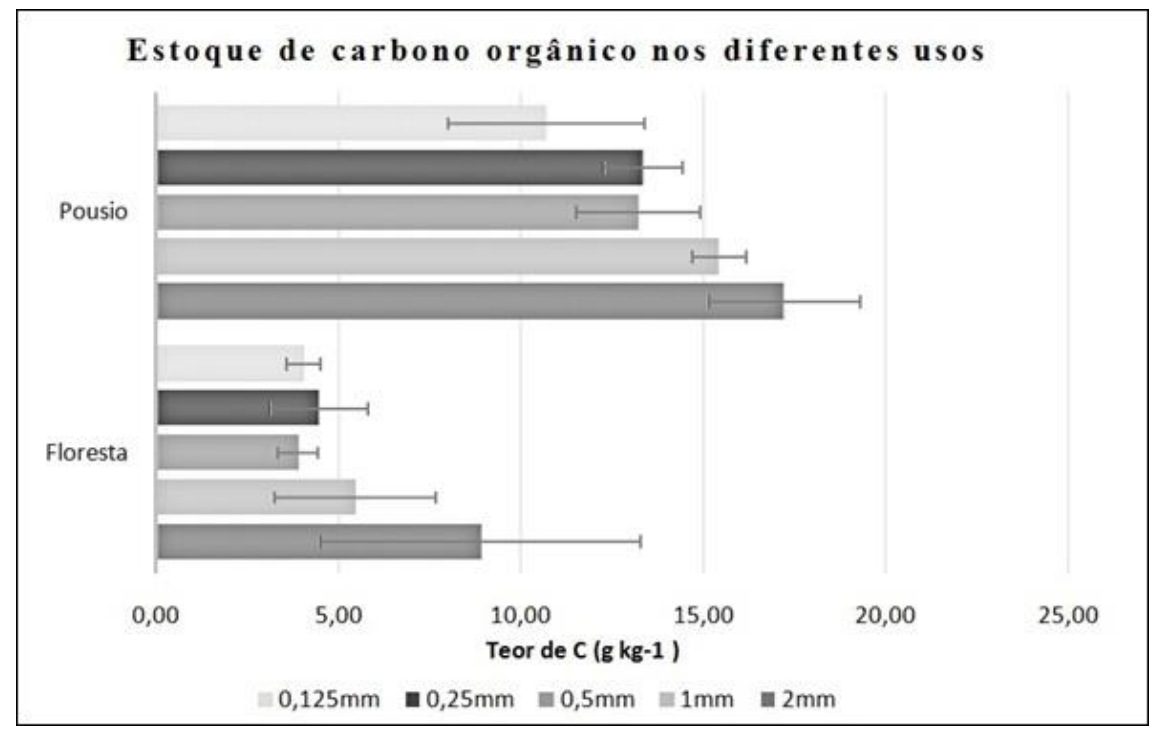

Figura 04 - Estoque de Carbono Orgânico por classe agregado em diferentes usos. 


\section{AVALIAÇÃO MACRO E MICROESTRUTURAL DE SOLOS SOB DIFERENTES USOS A PARTIR DE ANÁLISES FÍSICAS, MICROSCOPIA ELETRÔNICA E ESPECTROSCOPIA}

Diversos estudos demonstram que há correlação entre o conteúdo de carbono orgânico no solo e a estabilidade dos agregados em água (PODWOJEWSKI et al., 2011, OLIVEIRA et al., 2013). Os resultados apresentados acima permitem inferir que a matéria orgânica no solo, viva ou morta, exerce papel fundamental na agregação (DENEF et al., 2001, CASTRO FILHO et al., 2002) principalmente nos primeiros centímetros do horizonte superficial, o que ficou evidente principalmente em relação a formação de macroagregados. O pousio com gramíneas transmite deste modo uma boa macroagregação, o que leva aos bons resultados nos índices de agregação, porém, a resiliência e a manutenção deste ambiente são bastante questionáveis.

Os resultados do IEA representam uma média da agregação total (figura 5), sendo influenciados diretamente pelos dados de DMP e DMG, que por sua vez, podem ser influenciados pela matéria orgânica presentes no solo, que também interfere na distribuição de micro e macroagregados.

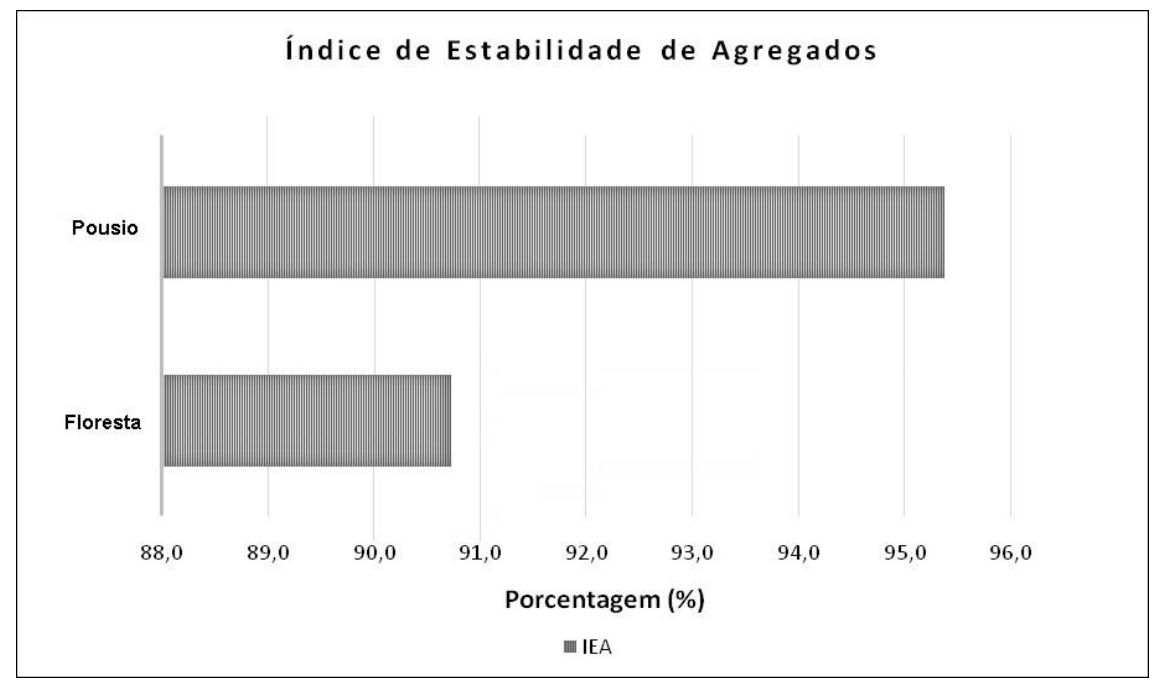

Figura 05 - Índice de Estabilidade dos Agregados do solo em água sob diferentes usos.

Demarchi et al. (2011), ao avaliarem a estabilidade dos agregados em diferentes sistemas de uso da terra, apresentaram os maiores valores de IEA para o pousio $(92,88 \%)$, enquanto que a mata nativa apresentou 88,44 $\%$. Os autores atribuem os maiores valores de IEA à classificação argilosa e ao teor de matéria orgânica encontrada.

Christensen (2001) afirma que além das interações entre os minerais, a interação destes com a matéria orgânica, constituindo complexos organominerais, afetam intensamente o tamanho dos agregados estáveis em água. Essa afirmação corrobora com os resultados apresentados, pois, quanto maior o tamanho dos agregados, maior o teor de Carbono orgânico, nos dois usos da terra (Figura 5).
Denef et al. (2001) destacam a importância dos macroagregados em controlar a estabilização do carbono e da matéria orgânica no solo. Knicker et al. (2005), ao analisarem um Cambissolo de floresta de Pinus, da região mediterrânea da Espanha, evidenciaram que as queimadas espontâneas frequentes provocaram aumento de $100 \%$ nos teores de Carbono e de Nitrogênio no horizonte A $(0-15 \mathrm{~cm})$, quando comparado com uma área próxima que não foi queimada.

Já a prática da queimada no pousio pode auxiliar no aumento do Carbono orgânico e de outros nutrientes ( $N, P, K, C a$ e $M g$ ) que favorecem a agregação e estruturação do solo. Entretanto, Centrini (2005) destaca que a absorção de nutrientes no solo por meio da queimada é muito efêmera, principalmente em 


\section{AVALIAÇÃO MACRO E MICROESTRUTURAL DE SOLOS SOB DIFERENTES USOS A PARTIR DE ANÁLISES FÍSICAS, MICROSCOPIA ELETRÔNICA E ESPECTROSCOPIA}

regiões de elevada distribuição pluviométrica anual.

DeBano (1988) destaca que grande parte da matéria orgânica queimada é volatilizada, sendo uma parte incorporada aos $5 \mathrm{~cm}$ superiores do solo e que age de forma "compactante", alterando a estrutura e impedindo a infiltração da água no solo, sendo este processo mais usual em solos mais arenosos. Ademais, Horton (1945), Bertoni e Lombardi Netto (2010) e Osmam (2014) destacam a fragilidade com que os solos ficam submetidos pela retirada da vegetação, dando início ao processo erosivo pelo Splash, resultando em sulcos, ravinas e voçorocas nas encostas.

As figuras 6 e 7 ilustram as análises realizadas por imagem a partir do Microscópio Eletrônico de Varredura (MEV). Os resultados mostram qualitativamente que a agregação do solo sob uso florestal é muito maior do que sob o uso do pousio, e que há uma predominância dos elementos $\mathrm{O}, \mathrm{Al}, \mathrm{Si}$, e $\mathrm{Fe}$ nos dois usos, onde, mineralogicamente, representam silicatos e óxidos de Ferro e Alumínio.

No solo sob floresta também foi possível identificar $\mathrm{C}$ e $\mathrm{K}$, enquanto que no pousio foi encontrado $\mathrm{P}$ e Ag. Assim, destacamse os seguintes valores para o uso florestal: Carbono (C) - 15\%, Oxigênio (O) - 56,07\%, Alumínio (Al) - 11,83\%, Sílica (Si) - 11,37\%, Potássio (K) - 0,40\% e Ferro (Fe) - 5,23\%. Já para o pousio: Carbono (C) $-5,80 \%$, Oxigênio (O) - 62,91\%, Alumínio (Al) - 9,42\%, Sílica (Si) 12,51\%, Fósforo (P) - 7,14\%, Ferro (Fe) - 4,61\% e Prata $(\mathrm{Ag})-3,41 \%$. Destaca-se a diferença na escala de frequência dos elementos químicos pelo EDS e que a as rachaduras visualizadas nas imagens não podem ser confundidas com a porosidade, pois as mesmas foram geradas em função do ressecamento da amostra. 
Brizzi et al.

AVALIAÇÃO MACRO E MICROESTRUTURAL DE SOLOS SOB DIFERENTES USOS A PARTIR DE ANÁLISES FÍSICAS, MICROSCOPIA ELETRÔNICA E ESPECTROSCOPIA

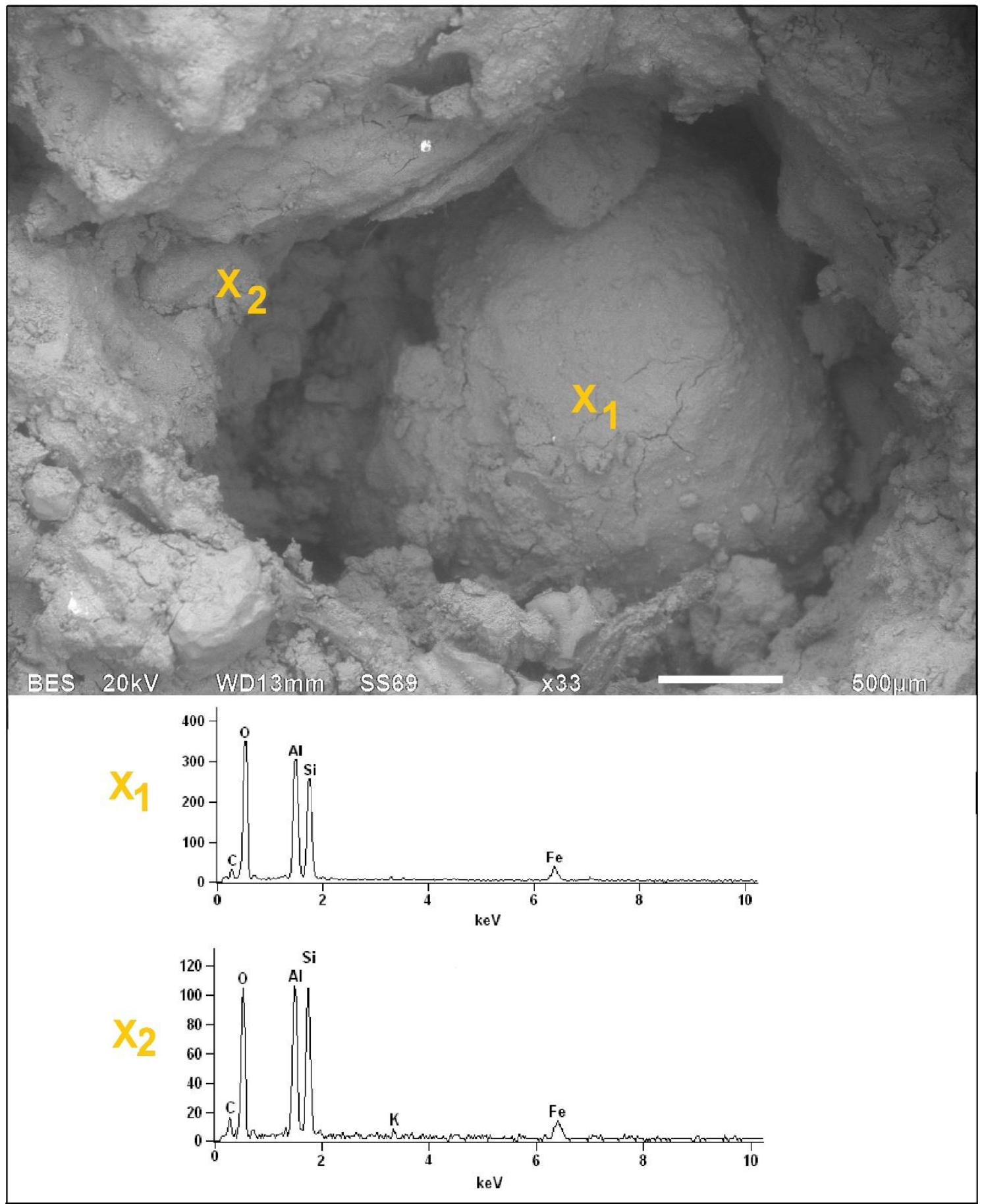

Figura 06 - Análise da estrutura do horizonte A do Cambissolo Háplico sob uso florestal no MEV e a identificação dos elementos químicos pelo EDS. 


\section{AVALIAÇÃO MACRO E MICROESTRUTURAL DE SOLOS SOB DIFERENTES USOS A PARTIR DE ANÁLISES FÍSICAS, MICROSCOPIA ELETRÔNICA E ESPECTROSCOPIA}
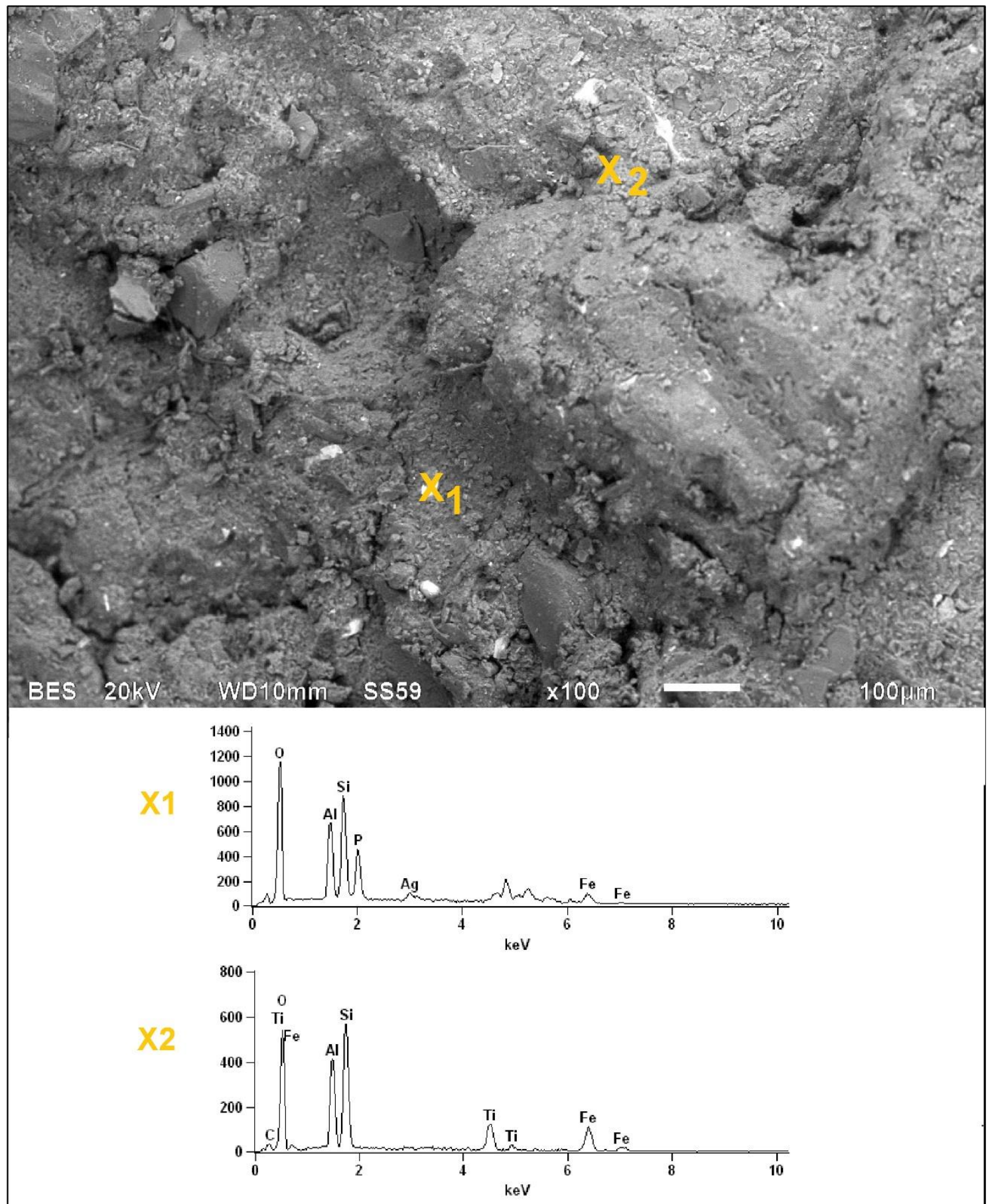

Figura 07 - Análise da estrutura do horizonte A do Cambissolo sob pousio no MEV e a identificação dos elementos químicos pelo EDS.

Enquanto que no uso florestal o solo apresenta maior indicativo de agregação (figura 6), no pousio (figura 7), percebe-se qualitativamente indicativo de maior cimentação da estrutura, de modo que possibilita identificar o solo como espécie de "maçaroca" ou "socado", que ajuda a deflagrar processos erosivos nas encostas (figura 8). Além disso, é mais fácil visualizar a microagregação sob uso florestal do que no pousio, sendo o primeiro identificado com um 


\section{AVALIAÇÃO MACRO E MICROESTRUTURAL DE SOLOS SOB DIFERENTES USOS A PARTIR DE ANÁLISES FÍSICAS, MICROSCOPIA ELETRÔNICA E ESPECTROSCOPIA}

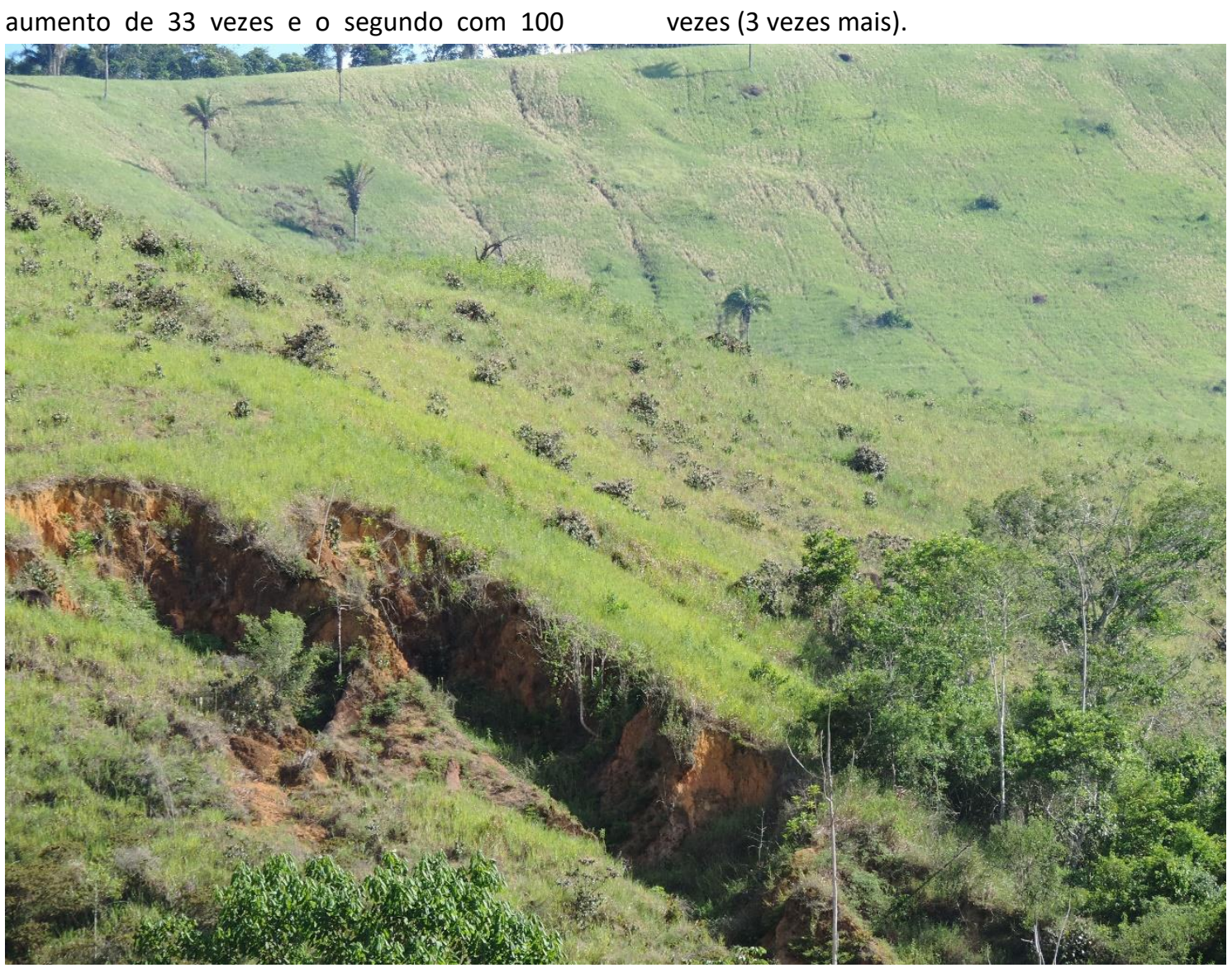

Figura 08 - Diferentes processos erosivos numa encosta na BHCC.

As imagens obtidas no MEV deste modo demonstraram ser o uso florestal mais correlacionado à boa microagregação dos horizontes superficiais dos solos avaliados, enquanto que o solo sob pousio apresentou uma microestrutura cimentada sugerindo forte antropização do horizonte, possivelmente por pé de arado.

\section{CONSIDERAÇÕES FINAIS}

De acordo com os objetivos propostos e os resultados apresentados, têm-se as seguintes conclusões:

1. Apesar de ter apresentado os melhores índices de agregação, o solo sob pousio apresentou uma microestrutura cimentada sugerindo forte antropização do horizonte, possivelmente por pé de arado, o que ficou evidenciado através da interpretação das imagens do MEV.

2. Os maiores valores encontrados nos índices de agregação no solo sob pousio podem refletir tanto a prática da queimada quanto a existência de uma ciclagem de nutrientes e uma deposição de serapilheira mais rápidas em relação ao ecossistema florestal, resultando em maiores valores de carbono orgânico em superfície, formação de macroagregados e aumento do IEA. Este aspecto, no entanto, não deve ser confundido com uma maior resiliência para o ambiente em relação ao uso florestal.

3. As análises no MEV possibilitaram detectar no solo sob uso florestal uma melhor microagregação nos horizontes superficiais em relação ao encontrado no solo sob pousio. Mesmo apresentando índices de agregação menores, a quantidade de carbono orgânico presente e possivelmente uma maior atividade biológica foram ambos determinantes para uma melhor microagregação.

4. Os resultados deste trabalho evidenciaram $o$ auxílio fornecido pela ferramenta MEV na compreensão dos 


\section{AVALIAÇÃO MACRO E MICROESTRUTURAL DE SOLOS SOB DIFERENTES USOS A PARTIR DE ANÁLISES FÍSICAS, MICROSCOPIA ELETRÔNICA E ESPECTROSCOPIA}

processos de agregação, sobretudo em relação à microagregação, possibilitando uma discussão mais ampla e profunda acerca do efeito de diferentes práticas de manejo e conservação dos solos em seus estados de agregação.

\section{REFERÊNCIAS}

AN, S.; MENTLER, A.; MAYER, H.; BLUM, W. E. $\mathrm{H}$. Soil aggregation, aggregate stability, organic carbon and nitrogen in different soil aggregate fractions under forest and shrub vegetation on the Loess Plateau, China. Catena, v. 81, p. 226233, 2010.

BERTOL, I.; GOBBI, E.; BARBOSA, F. T.; PAZFERREIRO, J.; GEGLER, L.; RAMOS, J. C.; WERNER, R. de S. Erosão hídrica em campo nativo sob diversos manejos: perdas de água e solo e de fósforo, potássio e amônio na água de enxurrada. Revista Brasileira de Ciência do Solo, v.35, p. 1421-1430, 2011.

BERTONI, J.; LOMBARDI NETO, F. Conservação do solo. São Paulo: Ícone, 2010.

BIGARELLA, J. J.; MOUSINHO, M. R.

Considerações a respeito dos terraços fluviais, rampas de colúvio e várzeas. Boletim Paranaense de Geografia (16/17): 153-198, Curitiba, 1965.

BRONICK, C. J.; LAL, R. Soil structure and management: A review. Geoderma. v. 124, p.322, 2005.

CARTER, M. R. Organic matter and sustainability. In: REES, B.C.; BALL, B.C.; CAMPBELL, C. D.; WATSON, C. A., eds. Sustainable management of soil organic. Wallingford, CAB International, 2001. p. 9-22.

CASTRO FILHO, C.; MUZILLI, O.; PODANOSCHI, A. L. Estabilidade dos agregados e sua relação com o teor de carbono orgânico num Latossolo Roxo Distrófico, em função de sistemas de plantio, rotações de culturas e métodos de preparo das amostras. Revista Brasileira de Ciência do Solo. v. 22, p. 527-538, 1998.

CASTRO FILHO, C.; LOURENÇO, A.; de F. GUIMARÃES, M.; FONSECA, I. C. B. Aggregate stability under different soil management systems in a red latosol in the state of Parana, Brazil. Soil and Tillage Research, v.65, n.1, p.4551, 2002.

CERTINI, G. Effects of fire on properties of forest soils: a review. Oecologia, v.143, p.1-10, 2005.

CHRISTENSEN, B. T. Physical fractionation of soil and structural and functional complexity in organic matter turnover. Europe Journal Soil Science. v. 52, p. 345-353, 2001.

COELHO NETTO, A. L. Hidrologia de encosta na interface com a geomorfologia. In: Guerra, A. J. T.; CUNHA, S. B. (Ed.). Geomorfologia: Uma Atualização de Bases e Conceitos. Rio de Janeiro: Bertrand Brasil, 2009. p.211-246.

DeBano, L. F. Effects of fire on chaparral soils in Arizona and California and postfire management implications. In: Berg, N., ed. FIRE AND WATERSHED MANAGEMENT: SYMPOSIUM PROCEEDINGS; 1988, October 16-28;

Sacramento, CA. Gen. Tech. Rep. PSW-109. Berkeley, CA: U.S. Department of Agriculture, Forest Service, Pacific Southwest Forest and Range Experiment Station: 55-62.

DEMARCHI, J. C.; PERUSI, M. C.; PIROLI, E. L. Análise de agregados da microbacia do Ribeirão São Domingos, Santa Cruz do Rio Pardo - SP, sob diferentes tipos de uso e ocupação. Revista Brasileira de Tecnologia Aplicada nas Ciências Agrárias, Guarapuava - PR, v.4, n.2, p.07-29, 2011.

DENEF, K.; SIX, J.; PAUSTIAN, K.; MERCKX, R. Importance of macroaggregate dynamics in controlling soil carbon stabilization: short-term effects of physical disturbance induced by drywet cycles. Soil Biology and Biochemistry, v. 33, p. 2145-2153, 2001.

EMBRAPA, Empresa Brasileira de Pesquisa Agropecuária. Mapa de reconhecimento de baixa intensidade dos Solos do estado do Rio de Janeiro. Ministério da Agricultura, pecuária e do Abastecimento, 2003.

Centro Nacional de Pesquisa de Solos. Manual de métodos de análise de solo. Rio de 


\section{AVALIAÇÃO MACRO E MICROESTRUTURAL DE SOLOS SOB DIFERENTES USOS A PARTIR DE ANÁLISES FÍSICAS, MICROSCOPIA ELETRÔNICA E ESPECTROSCOPIA}

Janeiro: EMBRAPA-CNPS. Documentos, 2011. $225 p$.

FATTET, M; FU, Y.; GHESTEM, M.; MA, W.; FOULONNEAU, M; NESPOULOUS, J. LE BISSONNAIS, Y.; STOKES, A. Effects of vegetation type on soil resistance to erosion: Relationship between aggregate stability and shear strength. Catena, v. 87, p. 60-69, 2011.

FERRARI, A. L. Evolução tectônica do Graben da Guanabara. Tese submetida ao Programa de Pós-Graduação em Geografia da USP. São Paulo, 2001. 381p. p.1-35; 64-89.

GROSBELLET, C.; VIDAL-BEAUDET, L.; CAUBEL, V.; CHARPENTIER, S. Improvement of soil structure formation by degradation of coarse organic matter. Geoderma, v. 162, p. 27-38, 2011.

HASUI, Y. A grande colisão pré-cambriana no sudeste brasileiro e a estruturação regional. São Paulo, UNESP, Geociências, v.29, n.2, p. 141-169, 2010.

HORTON, R.E. Erosional development of streams and their drainage basins: hydrophysical approach to quantitative morphology. Bulletin of the Geological Society

of America. Colorado, v. 56, p. 275-370, 1945.

ICMBIO. Plano de Manejo da APA de Cairuçu. 2004. Disponível em:

http://www.icmbio.gov.br/portal/component/c ontent/article?id=2240: apa-de-cairucu. Acesso em 10 de abril de 2017.

KEMPER, W. D.; ROSENAU, R. C. Aggregate stability and size distribution. In: KLUTE, A. (Ed.) Methods of soil analysis - Part I: Physical and minerological methods. Wisconsin: Madison, 1986.p.425-442.

JOHNSON, E. A. Fire and vegetation dynamics: studies from the North American boreal forest. Cambridge: Cambridge University Press, 129p. 1992.KIEHL, E. J. Manual de edafologia relações solo - planta. São Paulo: Agronômica Ceres, 1979.

KNICKER, H.; GONZÁLEZ-VILA, F.J.; POLVILLO, O.; GONZÁLEZ, J.A.; LMENDROS, G. Wildfire induced alterations of the chemical composition of humic material in a Dystric Xerochrept under a Mediterranean pine forest (Pinus pinaster Aiton). Soil Biology and Biochemistry, v.37, p.701-718, 2005.

MALISKA, A, M. Microscopia Eletrônica De Varredura.

http://www.usp.br/nanobiodev/wpcontent/uploads/MEV Apostila.pdf Acesso em 17 de abril de 2017.

MENDES, C. A. R.; MAHLER, C. F.; ANDRADE, A. G. de. Erosão superficial em argissolo amarelo sob cultivo perene e com pousio florestal em área de relevo montanhoso. Revista Brasileira de Ciência do Solo, v.35, n.4, Viçosa, 2011. MORGAN, R. P. C. Soil erosion and conservation. Inglaterra: Blackwell Publishing, 2005.

ASSOCIAÇÃO BRASILEIRA DE NORMAS TÉCNICAS - ABNT. Solo - Determinação dos ensaios de densidade relativa dos grãos sólidos NBR 6508, 1984a, ABNT, Rio de Janeiro.

Solo - Análise Granulométrica. NBR 7181, 1984b, ABNT, Rio de Janeiro.

OLIVEIRA, J. G. R.; FILHO TAVARES, J.; BARBOSA, G. M. C. Qualidade física do solo das trilhas do parque estadual do Cerrado - PR. Ciências Agrárias, Londrina, v. 34, n. 4, p. 1715-1722, 2013.

OSMAN, K. T. Soil degradation, conservation and remediation. Bangladesh: Springer, 2014.

PODWOJEWSKI, P.; POULENARD, J.; NGUYET, M. L.; ROUW, A.; NGUYEN, V. T.; HA PHAM, Q.; TRAN, D. C. Climate and vegetation determine soil organic matter status in an alpine innertropical soil catena in the Fan Si Pan Mountain, Vietnam. Catena, v. 87 p. 226-239, 2011.

PUIG, H. A floresta tropical úmida. Tradução de Maria Leonor Frederico Rodrigues Loureiro. São Paulo: UNESP: Imprensa Oficial do Estado de São Paulo; França: Institut de Rechérche pour le Developpement, 2008. p. 55-105; 195-203; 253-297; 329-431.

SANTOS, C. A.; SOBREIRA, F. G.; COELHO NETO, A. L. Comportamento hidrológico superficial e 


\section{AVALIAÇÃO MACRO E MICROESTRUTURAL DE SOLOS SOB DIFERENTES USOS A PARTIR DE ANÁLISES FÍSICAS, MICROSCOPIA ELETRÔNICA E ESPECTROSCOPIA}

erodibilidade dos solos da região de Santo

Antônio do Leite, Distrito de Ouro Preto - MG.

Revista Escola de Minas, v.55, p.285-290,

2002.

SANTOS, R. D.; LEMOS, R. C.; SANTOS, H. G.;

KER, J. C; ANJOS, L. H. C. Manual de descrição e coleta de solo no campo. Viçosa, MG:

Sociedade Brasileira de Ciência Do Solo, 2005.

100p.

SILVA, I. F.; MIELNICZUK, J. Avaliação do estado de agregação do solo afetado pelo uso agrícola. Revista Brasileira de Ciência do Solo, v.21, p.313-319, 1997.

SILVA, I. F.; MIELNICZUK, J. Sistemas de cultivo e características do solo afetando a estabilidade de agregados. Revista Brasileira de Ciência do Solo, v.22, p.311-317, 1998.

SHUKLA, M. K.; LAL, R.; EBINGER, M. Determining soil quality indicators by factor analysis. Soil and Tillage Research, 87:194-204, 2006.

TISDALL, J. M.; OADES, J. M. Organic matter and water-stable aggregates in soils. J. Soil Science, 33:141-163, 1982.

TRIOLA, M. F. Introdução à Estatística. Rio de Janeiro: LTC, 2008.

WENDLING, B.; JUCKSCH, l; MENDONCA, E. de S. ; NEVES, J. C. L. Carbono orgânico e estabilidade de agregados de um Latossolo Vermelho sob diferentes manejos. Pesquisa Agropecuária brasileira. Brasília, v.40, n.5, p.487-494, 2005.

YODER, R. E. A direct method of aggregate analysis of soils and a study of the physical nature of erosion losses. Journal American Society Agronomy. v. 28, p. 337-351. 1936. 